\title{
Association of cognitive impairment and elderly mortality: differences between two cohorts ascertained 6-years apart in China
}

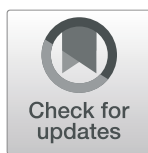

Jun Duan ${ }^{1,2 \dagger}$, Yue-Bin Lv² ${ }^{2 \dagger}$ Xiang Gao ${ }^{3}$, Jin-Hui Zhou ${ }^{2}$, Virginia Byers Kraus ${ }^{4}$, Yi Zeng ${ }^{5,6}$, Hong Su $^{1^{*}}$ and Xiao-Ming Shi ${ }^{2^{*}}$

\begin{abstract}
Background: Cognitive impairment is a major contributor to mortality among the elderly. However, the relationship between cognitive impairment evaluated by educational levels and mortality and the trend between cognitive impairment and mortality with time are unclear. We aim to evaluate the differences in associations of cognitive impairment, taking the stratification by educational levels into account, with all-cause mortality and further explore the relationship of cognitive impairment with mortality in different age and sex groups in two cohorts ascertained 6 years apart in China.
\end{abstract}

Methods: A total of 13,906 and 13,873 Chinese elderly aged 65 years and older were included in the 2002-2008 and 2008-2014 cohorts from the Chinese Longitudinal Healthy Longevity Survey (CLHLS). Mortality data was ascertained from interviews with family members or relatives of participants. Cognitive function, evaluated by the Mini-Mental State Examination (MMSE), were defined by different cut-offs taking educational background into account. Cox models were used to explore the relationship of cognitive impairment with mortality.

Results: For the 2002-2008 and 2008-2014 cohorts, 55,277 and 53,267 person-years were followed up, and the mean (SD) age were 86.5 (11.6) and 87.2 (11.3) years, respectively. Compared to normal cognition, cognitive impairment was independently associated with higher mortality risk after controlling for potential confounders, with hazard ratios (HRs) of 1.32 (95\% confidence interval [Cl], 1.25-1.39) in 2002-2008 cohort and 1.26 (95\% Cl, 1.19-1.32) in 2008-2014 cohort, stratified by educational levels. The trend of cognitive impairment with all-cause mortality risk decreased from 2002 to 2008 to 2008-2014 cohort, while no significant interaction of cognitive impairment with cohort for all-cause mortality was observed. The associations of cognitive impairment and mortality were decreased with age in the two cohorts.

Conclusions: Cognitive impairment evaluated by different cut-offs were associated with increased risk of mortality, especially among those aged 65-79years in the two cohorts; this advocates that periodic screening for cognitive impairment among the elderly is warranted.

Keywords: Aging, Cohort study, Cognitive impairment, Oldest old, Mortality

\footnotetext{
* Correspondence: suhong5151@sina.com; shixm@chinacdc.cn

†Jun Duan and Yue-Bin LV contributed equally to this work.

${ }^{1}$ Department of Epidemiology and Health Statistics, School of Public Health,

Anhui Medical University, 81 Meishan Road, Hefei 230032, Anhui Province,

China

${ }^{2}$ National Institute of Environmental Health, Chinese Center for Disease

Control and Prevention, \#7 Panjiayuan Nanli, Chaoyang, Beijing 100021,

China

Full list of author information is available at the end of the article
}

(c) The Author(s). 2020 Open Access This article is distributed under the terms of the Creative Commons Attribution 4.0 International License (http://creativecommons.org/licenses/by/4.0/), which permits unrestricted use, distribution, and reproduction in any medium, provided you give appropriate credit to the original author(s) and the source, provide a link to the Creative Commons license, and indicate if changes were made. The Creative Commons Public Domain Dedication waiver (http://creativecommons.org/publicdomain/zero/1.0/) applies to the data made available in this article, unless otherwise stated. 


\section{Introduction}

Cognitive impairment is a major risk factor for poor health in the growing population of elders worldwide [1-3]. It imposes a heavy burden on public health and is associated with shortened life expectancy. The prevalence of mild cognitive impairment aged 65 years or older in China was about $20.8 \%$ in 2014 [4]; more than half of these individuals progresses to dementia within 5 years [5]. As China, the world's largest developing country quickly transitions into an aging society, it was reported that the mortality attributable to dementia in China increased from 1.6 million in 1990 to 2.3 million in 2016 [6], which can profoundly impact Chinese elderly health-related quality of life and longevity.

Although a number of epidemiological studies have reported on a cognitive impairment-mortality relationship $[3,7]$, most of them concerned the association of cognitive impairment and mortality risk of elders in high-income contries [8-12]. In upper-middle income countries such as China, several studies have indicated that baseline cognitive impairment increases the risk of all-cause mortality $[13,14]$. Earlier studies have found that education is strongly related with cognitive performance [15]. In China, illiteracy is still widely prevalent, particularly among the elderly population [16]. In previous studies, the prevalence of cognitive impairment was different for entire cohort according to the cut-offs of education, which is higher than reported previously in China [16]. Therefore, it's necessary to consider cognitive impairment, stratified by different cut-offs taking educational background into account when we investigate the relationship between cognitive impairment and mortality in the elderly population. In addition, women have a higher risk of cognitive impairment, while a lower risk of death may lead to gender differences. The relationship between cognitive impairment and mortality risk have been extensively reported and the results were inconsistent [13, 17]. Moreover, studies are limited that include large sample sizes, national representation, different age groups in the oldest old (aged 80 and older).

Prior studies have explored the association of cognitive impairment and all-cause mortality risk with long-term follow-up (14 years and 20 years) $[13,17]$, but have rarely studied the impacts of change in medical, demographic and social factors over time on the association between cognitive impairment and mortality. It has been reported that annual mortality among the oldest old was substantially declined between 0.2 and $1.3 \%$ in 1998-2008 compared with the participants of the same age born before 10 years, but cognitive impairment increased annually between 0.7 and $2.2 \%$ in the past ten years [18]. Therefore, it is unclear whether the impact of cognitive impairment on mortality has changed with the passage of time.

The present study aims to examine and compare the relationship between cognitive impairment, stratified by different cut-offs taking educational background into account, and mortality using two cohort studies conducted in 2002-2008 and 2008-2014. Moreover, subgroup analyses were further conducted among different sex and age groups to identify susceptible populations in 20022008 and 2008-2014.

\section{Materials and methods}

\section{Study design and participants}

The Chinese Longitudinal Healthy Longevity Survey (CLHLS) was a nationwide survey that randomly selected half of the cities and counties in 23 provinces of China, and recruited participants aged 65 years and older. A more detailed description of the CLHLS has been published elsewhere [19].

The protection of human subjects for the CLHLS was approved by the Ethics Committees. All participants or their legal representatives signed written consent forms to participate in the baseline and follow-up surveys.

\section{Assessment of cognitive impairment and mortality}

The present study evaluated baseline cognitive status of two cohorts, ascertained in 2002 and 2008, using the same scale of cognitive function. Cognitive impairment was evaluated using the Mini-Mental State Examination (MMSE), a widely used cognitive test [20] and adapted into the Chinese language based on the international standard of MMSE questionnaire, and carefully tested by previous pilot survey interviews [21]. The total MMSE score ranges from 0 to 30 within 6 dimensions: orientation, registration, attention, language, memory, and visual construction skills. Three methods were used to define cognitive impairment: (1) $\geq 24,18-23$, and $<18$ were used to define normal cognition (reference), mild cognitive impairment and serious cognitive impairment $[22,23] ;(2)<18$ was used to define cognitive impairment for participants who didn't receive any formal education, < 21 for participants who received 6 years of education or less, and $<25$ for participants who received more than 6 years of education [16, 24]; (3) $\geq 24$ and $<23$ were used to define normal cognition (reference) and cognitive impairment $[22,23]$.

The main outcome was all-cause mortality occurring during the follow-up survey in 2002-2008 and 20082014, with followed up every 3 years respectively. Each cohort was followed for 6 years to quantify mortality and the mortality date. Mortality date was ascertained from interviews with family members or relatives of participants [18]. The cause-specific mortality was not involved in this study because (1) many of the elderly died at home rather than in medical institutions where cause of mortality might be recorded, and (2) mortality surveillance systems are uncertain in many survey fields. 


\section{Assessment of potential confounding variables}

A number of variables were collected through a face-toface standardized questionnaire, including demographic characteristics, economic status, lifestyles, health conditions and medical services.

Marital status was classified into unmarried and married. Education level was classified as no formal education, elementary school graduate (1-6 years of education), and high school graduate ( $>6$ years of education). Region was defined as: urban, rural and suburban. Exercise was categorized into yes or no. Housework and reading were divided into 3 categories: never, sometimes, and often. Binary variables were defined to assess current status of smoking, drinking, depression and disability in six activities of daily living (ADL) including dressing, bathing, using the toilet, getting in/out of a bed or chair and feeding. An ADL impairment was defined as a elder' s response of "needs help" to at least one or more of activities associated with one of the six items. Participants with systolic blood pressure $\geq 140 \mathrm{mmHg}$ or diastolic blood pressure $\geq 90 \mathrm{mmHg}$ were considered hypertensive. Selfreported history of heart disease and stroke were also collected. According to the survey, we assessed the level of medical care by whether the participant was receiving adequate medical care at present? (Yes or No)" and on the basis of the payor of the medical costs (public medicare or not)". We assessed economic status by asking, "are all financial sources enough for your life?", "do you have a pension?" and "how is your life compared with other local people? (richer, similar and poorer)".

\section{Statistical analysis}

We divided the elderly into 3 groups by the conventional MMSE cut-off points for 2002-2008 and 2008-2014. Mean and standard deviation were summarized for continuous variables, frequency and percentage for categorical variables. Comparisons between the elderly were conducted using the chi-square test for categorical variables, Kruskal-Wallis test for continuous variables.

Kaplan-Meier analysis was used to draw the survival curves stratified by MMSE score, compared by the logrank test. We used the Cox proportional hazards models and the important confounders were identified by previous studies [13, 17]. Less than $1.3 \%$ for all independent variables had missing values, and due to such low missing rates, we deleted the cases with missing values, and the results have no significant difference compared to those with imputation [18].

Hazards ratio (HR) and 95\% confidence intervals (CIs) were estimated with the construction of Cox proportional hazards models: the crude model was model 1; age and sex were adjusted in model 2; marital status, living alone, exercise, alcohol consumption, and smoking status were further adjusted in model 3; ADL and depression were further adjusted in model 4; and medical care and economic status were further adjusted in model 5 . We pooled data from the 2002 cohort and the 2008 cohort and a variable that was assigned a value of 0 in the 2002 cohort and 1 in the 2008 cohort was included in the Cox proportional hazards model [25]. We tested the interaction between each cohort and cognitive impairment and explored whether the influence of cognitive impairment on mortality decreases over time. For subgroup analyses, the elderly were stratified by age group $(65-79,80-89,90-99$, and $\geq$ 100 ) and sex (male and female) in model 5 . Interactions of baseline cognitive impairment with age group and sex on mortality risk were explored.

We cannot measure long-term progression about cognitive function on mortality. Previous studies demonstrated that both cognitive impairment and disability were independent of adverse impact on mortality [26, 27]. Therefore, the interaction synergistic effects between ADLs and cognitive impairment were tested in the models.

The following sensitivity analyses were conducted to check the robustness of the primary results: (1) removed the participants lost to follow-up to examine possible attrition bias; (2) excluded survival time less than 1 year due to the possibility that disease status in the last year of life could have affected the risk effects; (3) additionally adjusting for place of residence, dietary habits, hypertension, self-reported heart disease and stroke.

Data analysis was conducted using $\mathrm{R}$ version 3.3.1. All statistical tests were 2-sided, and statistical significance was judged by $P$-values $<0.05$.

\section{Results}

\section{Baseline characteristics}

A total of 13,906 and 13,873 elderly aged 65 and older participated in the baseline survey in 2002 and 2008 . The mean (SD) age of the elderly were 86.52 (11.63) and 87.22 (11.33) years in the two cohorts. A total of 55,277 person-years were documented in the 2002-2008 cohort, and 53,267 in the 2008-2014 cohort. Characteristics of the elderly who survived, died or lost to follow-up are displayed in Fig. 1. Participants with cognitive impairment tended to be older; female; unmarried; less likely to smoke, drink and live alone; have lower baseline $\mathrm{ADL}$ and depression; have higher rates of inadequate medical care and no pension. The baseline conditions of the two cohorts were similar (Table 1).

\section{Relationship between cognitive impairment and all-cause mortality}

Additional file 1: Figure S1 shows the Kaplan-Meier survival curve by different categories of MMSE-based cognitive impairment. Significant differences were revealed by the log-rank test in the 3 groups (cognitive normal, mild cognitive impairment and serious cognitive impairment; 


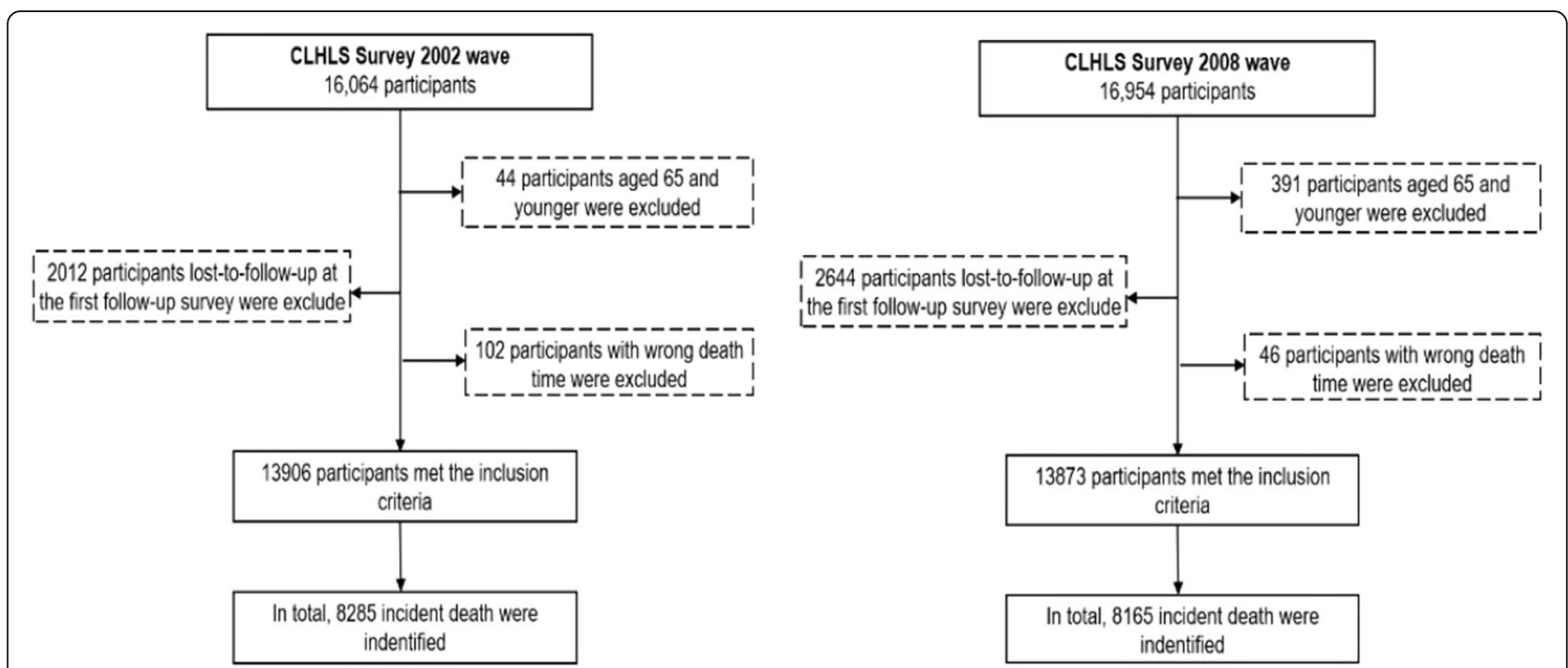

Fig. 1 Flowchart of the study population, the Chinese Longitudinal Healthy Longevity Survey (CLHLS) form 2002-2008 (Left) and 2008-2014 (Right)

$P<0.001)$ among the two cohorts. Table 2 shows that risk of mortality increased in parallel with a decrease in MMSE scores. In the crude model, the elderly with cognitive impairment (MMSE < 18) had an elevated risk of all-cause mortality compared to the other MMSE categories in 2002-2008 (MMSE < 18 [crude HR, 3.56; 95\% CI, 3.393.74]) and 2008-2014 ([crude HR, 3.25; 95\% CI, 3.10$3.41]$ ). After adjusting for sex and age (model 2), demographic characteristics (model 3), functional status (model 4), and medical care and economic status (model 5), similar associations were found between cognitive impairment and mortality in the two cohorts. In the fully adjusted model, participants with MMSE scores indicative of cognitive impairment had increased all-cause mortality risk compared with the participants with normal cognition: for 2002-2008 mild cognitive impairment HR 1.28 (95\% CI 1.20-1.37), severe cognitive impairment HR 1.48 (95\% CI 1.39-1.57); for 2008-2014 mild cognitive impairment HR 1.20 (95\% CI 1.12-1.28), severe cognitive impairment HR 1.32 (95\% CI 1.25-1.41) (Table 2). When cognitive impairment was stratified by educational levels, cognitive impairment was independently associated with higher mortality risk, with hazard ratios (HRs) of 1.32 (95\% confidence interval [CI], 1.25-1.39) in 2002-2008 cohort and 1.26 (95\% CI, 1.19-1.32) in 2008-2014 cohort, compared to normal cognition. Similar results were obtained when cognitive impairment was defined by MMSE $<24$ or considering education level in both cohorts.

The association of cognitive impairment with all-cause mortality over the time was decreased comparing the 2002-2008 cohort to the 2008-2014 cohort. However, no significant interaction of cognitive impairment for all-cause mortality among two cohorts was significant (Table 3, $P>0.05$ for interaction).

\section{Subgroup analysis}

Lower MMSE score was consistently associated with elevated risk of mortality both in 2002-2008 and 2008-2014. The analysis stratified by sex revealed that cognitive impairment was associated with increased risk of all-cause mortality in females compared to males in 2002-2008 with HR 1.35 (95\% CI 1.24-1.46) and in 2008-2014 HR 1.20 (95\% CI 1.10-1.32). However, an interaction of cognitive impairment with sex for all-cause mortality was not observed (Table 3, $P>0.05$ for interaction).

Compared to those with normal cognition, MSSE scores $>24$, the younger elderly (65-79 years old) with cognitive impairment had higher risk of all-cause mortality, respectively in the two cohorts (Table 3).

Both cognitive impairment and ADL impairment have been considered important risk factors associated with elderly mortality. During the aging process, cognitive impairment and ADL impairment often co-exist and closely interact (Additional file 1: Table S1, S2).

\section{Sensitivity analysis}

Among two cohorts, there was almost no change in the association between cognitive impairment and all-cause mortality after excluding participants lost to follow-up or with survival time less than 1 year. The association was still robust after further adjustment for potential confounders (Additional file 1: Table S3, S4).

\section{Discussion}

In this large-scale prospective analysis we explored the association of cognitive impairment and all-cause mortality based on two Chinese cohorts with 6 years each of follow-up. Firstly, cognitive impairment evaluated by MMSE score was closely related with an increased risk 


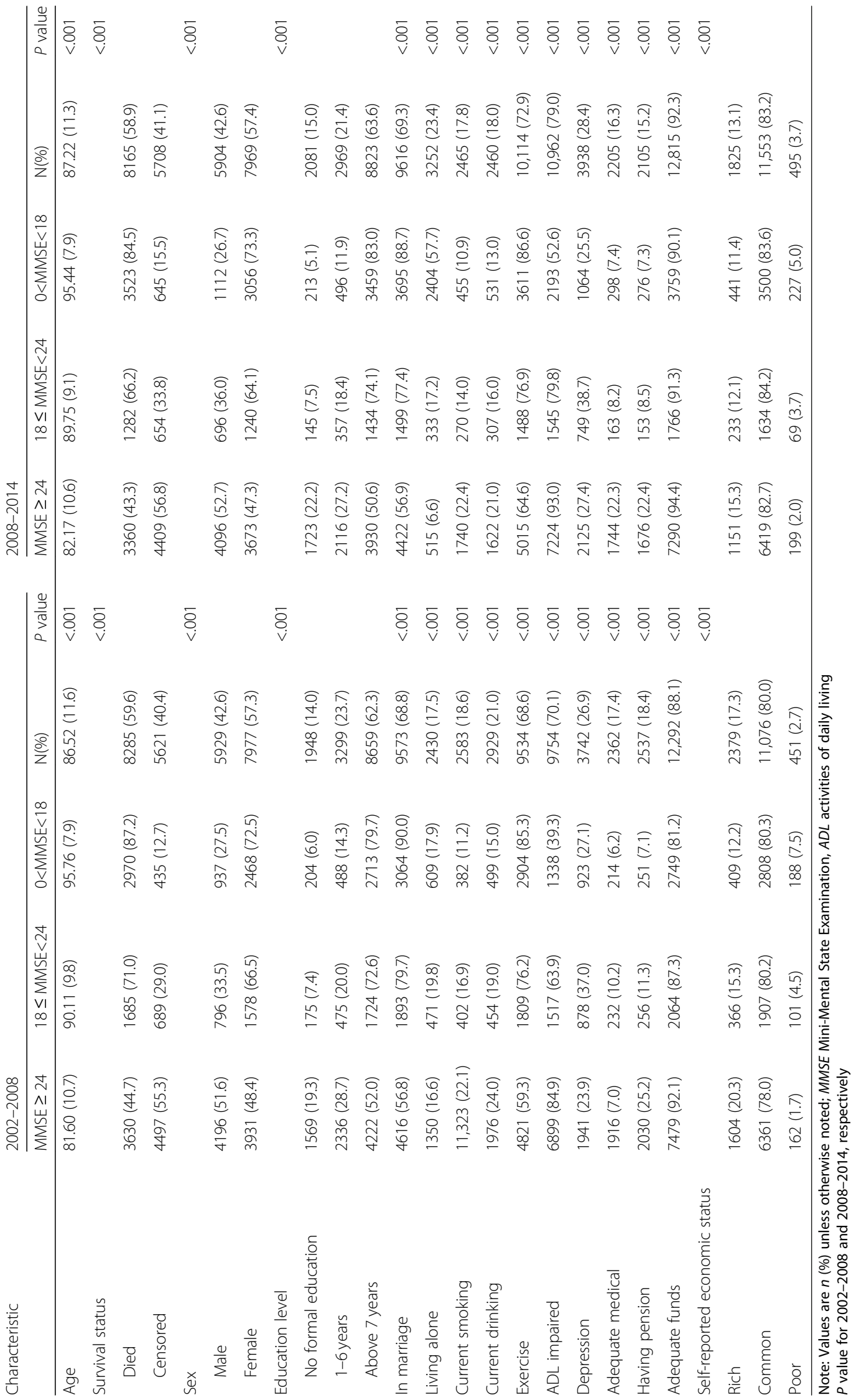


Table 2 Hazard ratios $(95 \% \mathrm{Cl})$ for all-cause mortality according to baseline MMSE Score

\begin{tabular}{|c|c|c|c|c|c|c|}
\hline \multirow[t]{2}{*}{ MMSE Score } & \multirow[t]{2}{*}{ Death } & \multicolumn{5}{|c|}{ Hazard Ratio(95\% Cl) } \\
\hline & & Model1 & Model2 & Model3 & Model4 & Model5 \\
\hline \multicolumn{7}{|l|}{$2002-2008$} \\
\hline Cognitive impairment by Education & $5069(49.9)$ & 1(Reference) & 1(Reference) & 1(Reference) & 1 (Reference) & 1(Reference) \\
\hline MMSE by Education & $3216(85.7)$ & $2.88(2.75,3.01)$ & $1.49(1.41,1.56)$ & $1.44(1.37,1.51)$ & $1.33(1.26,1.40)$ & $1.32(1.25,1.39)$ \\
\hline MMSE 24 to 30 & $3630(45.3)$ & 1(Reference) & 1(Reference) & 1(Reference) & 1 (Reference) & 1(Reference) \\
\hline MMSE $<24$ & $4655(81.8)$ & $2.85(2.72,2.97)$ & $1.54(1.47,1.62)$ & $1.48(1.41,1.56)$ & $1.39(1.32,1.46)$ & $1.38(1.31,1.45)$ \\
\hline MMSE 24 to 30 & $3630(45.3)$ & 1(Reference) & 1(Reference) & 1(Reference) & 1 (Reference) & 1(Reference) \\
\hline MMSE 18 to 23 & $1685(71.6)$ & $2.12(2.00,2.25)$ & $1.38(1.30,1.47)$ & $1.34(1.26,1.42)$ & $1.29(1.21,1.37)$ & $1.28(1.20,1.37)$ \\
\hline MMSE $<18$ & $2970(87.4)$ & $3.56(3.39,3.74)$ & $1.69(1.60,1.79)$ & $1.62(1.53,1.72)$ & $1.48(1.40,1.58)$ & $1.48(1.39,1.57)$ \\
\hline \multicolumn{7}{|l|}{ 2008-2014 } \\
\hline Cognitive impairment by Education & $4454(47.1)$ & 1(Reference) & 1(Reference) & 1(Reference) & 1 (Reference) & 1(Reference) \\
\hline MMSE by Education & $3711(84.2)$ & $2.80(2.68,2.92)$ & $1.53(1.45,1.60)$ & $1.37(1.30,1.44)$ & $1.29(1.22,1.36)$ & $1.26(1.19,1.32)$ \\
\hline MMSE 24 to 30 & $3630(45.3)$ & 1(Reference) & 1(Reference) & 1(Reference) & 1 (Reference) & 1(Reference) \\
\hline MMSE $<24$ & $4805(79.2)$ & $2.73(2.61,2.86)$ & $1.52(1.44,1.59)$ & $1.37(1.30,1.44)$ & $1.30(1.23,1.37)$ & $1.27(1.20,1.34)$ \\
\hline MMSE 24 to 30 & $4409(43.4)$ & 1(Reference) & 1(Reference) & 1(Reference) & 1 (Reference) & 1(Reference) \\
\hline MMSE 18 to 23 & $1282(66.2)$ & $1.92(1.80,2.05)$ & $1.30(1.22,1.39)$ & $1.25(1.17,1.34)$ & $1.22(1.14,1.30)$ & $1.20(1.12,1.28)$ \\
\hline$M M S E<18$ & $3523(85.1)$ & $3.25(3.10,3.41)$ & $1.65(1.56,1.74)$ & $1.46(1.38,1.55)$ & $1.36(1.28,1.45)$ & $1.32(1.25,1.41)$ \\
\hline
\end{tabular}

Note: The values of cognitive impairment are $\mathrm{n}$ and mortality rate in brackets(\%); $\mathrm{Cl}$ confidence interval, MMSE Mini-Mental State Examination, $A D L$ activities of daily living. Cognitive impairment by Education: $<18$ was used to define cognitive impairment for participants who didn't receive any formal education, $<21$ for participants who received 6 years of education or less, and $<25$ for participants who received more than 6 years of education

*Hazard ratio $(95 \% \mathrm{Cl})$ was calculated from Cox models

Model1: Unadjusted

Model2: Age + sex

Model3: Model2 + Marry+Living alone+Educational level + Exercise+Smoke+Drink

Model4: Model3 + ADL + Depression

Model5: Model4 + Medical service + Economic status

of all-cause mortality and the risk effect of cognitive impairment on mortality was lower by the cut-off of education level. Secondly, the association of cognitive impairment with risk of all-cause mortality was lower in 2008-2014 versus 2002-2008, but there were no significant interaction of cognitive impairment for all-cause mortality among two cohorts between them. Thirdly, the association of cognitive impairment with all-cause mortality was reduced with age in both cohorts.

A relationship between cognitive impairment and elevated risk of mortality in elders has been reported consistently by epidemiological studies. The MMSE as a general measure of cognitive impairment has been an important predictor of all-cause mortality $[28,29]$. To date, although few studies have explored the relationship between cognitive impairment and all-cause mortality in China [30-32], the existing results support our conclusion that cognitive impairment is independently associated with an increased risk of death in the elderly [13, 14]. Moreover, MMSE scores are known to be influenced by education [16]. The risk of cognitive impairment evaluated by MMSE score, stratified by education was lower than used by the same cutoff point as in Western countries. The effect might be overestimated due to the illiterate or less educated in
China. Thus, in the near future, as the level of education increases, it is possible that we will be able to re-evaluate by a certain cut-off.

This study discovered that the association of cognitive impairment with the risk value of all-cause mortality has declined among the Chinese elderly in 2008-2014 compared to 2002-2008 and an interaction of cognitive impairment for all-cause mortality among two cohorts was not significant. China has achieved medical insurance coverage for all people since China's medical reform in 2008. Governments at all levels are increasingly investing in medical reform, such as medical technology and equipment [33]. These benefits might be expected to play a part in reducing mortality from all causes in the later cohort.

Previous studies on sex differences in the association of cognitive impairment with all-cause mortality have been inconsistent [13, 34]; this might be attributed to regional differences and insufficient sample size for analysis. An et al. indicated that males had a higher risk than females, which might be attributed to an undesirable lifestyle among men, such as smoking and drinking [17]. Conversely, Kirsten found that women perform worse than men with respect to lifelong subnormal cognitive functioning or emotional disorders [22]. However, there are 
Table 3 The subgroup analyses of hazard ratios (95\% Cl) for all-cause mortality according to baseline MMSE score

\begin{tabular}{|c|c|c|c|c|c|}
\hline & MMSE & $2002-2008$ & P-interaction & 2008-2014 & $P$-interaction \\
\hline \multirow[t]{3}{*}{ Cohort } & & & I & & $P=0.254$ \\
\hline & MMSE 18 to 23 & $1.28(1.20,1.37)$ & & $1.20(1.12,1.28)$ & \\
\hline & MMSE $<18$ & $1.48(1.39,1.57)$ & & $1.32(1.25,1.41)$ & \\
\hline Sex & & & $P=0.663$ & & $P=0.810$ \\
\hline \multirow[t]{2}{*}{ Male } & MMSE 18 to 23 & $1.26(1.14,1.39)$ & & $1.20(1.08,1.34)$ & \\
\hline & MMSE $<18$ & $1.42(1.29,1.57)$ & & $1.23(1.11,1.36)$ & \\
\hline \multirow[t]{2}{*}{ Female } & MMSE 18 to 23 & $1.31(1.21,1.42)$ & & $1.21(1.10,1.32)$ & \\
\hline & MMSE $<18$ & $1.51(1.40,1.63)$ & & $1.40(1.29,1.51)$ & \\
\hline Age & & & $P<.001$ & & $P<.001$ \\
\hline \multirow[t]{2}{*}{$65-79$} & MMSE 18 to 23 & $1.41(1.16,1.72)$ & & $1.63(1.29,2.06)$ & \\
\hline & MMSE $<18$ & $2.15(1.64,2.83)$ & & $1.79(1.33,2.41)$ & \\
\hline \multirow[t]{2}{*}{$80-89$} & MMSE 18 to 23 & $1.27(1.14,1.42)$ & & $1.17(1.04,1.33)$ & \\
\hline & MMSE $<18$ & $1.75(1.56,1.97)$ & & $1.33(1.17,1.52)$ & \\
\hline \multirow[t]{2}{*}{ 90-99 } & MMSE 18 to 23 & $1.26(1.14,1.40)$ & & $1.16(1.05,1.29)$ & \\
\hline & MMSE $<18$ & $1.44(1.31,1.59)$ & & $1.39(1.27,1.53)$ & \\
\hline \multirow[t]{2}{*}{$100+$} & MMSE 18 to 23 & $1.09(0.97,1.24)$ & & $0.94(0.81,1.09)$ & \\
\hline & MMSE<18 & $1.29(1.16,1.43)$ & & $1.11(0.99,1.24)$ & \\
\hline
\end{tabular}

Note: $\mathrm{Cl}$ confidence interval, MMSE Mini-Mental State Examination

Reference: MMSE 24 to 30

*Hazard ratio $(95 \% \mathrm{Cl})$ was calculated from cox models after adjust age, sex, marry, living alone, educational level, exercise, smoke, drink, ADL, depression, medical service and economic status

also studies that reported no sex-specific differences [13]. Similarly, we did not find sex differences between baseline cognitive impairment and all-cause mortality. It is necessary for further analyses to explore possible different patterns of mortality among sexes with cognitive impairment.

Many population-based studies have reported that cognitive impairment was strongly associated with subsequent mortality in the elderly [22, 35, 36]. Our study was consistent with previous findings and further demonstrated that the association of cognitive impairment with all-cause mortality was more pronounced among younger elderly in two Chinese cohorts. One possible reason that cognitive impairment of younger elderly poses a greater risk of mortality may be due to their ability to develop cognitive impairment faster compared to the oldest old, thus triggering a greater risk of mortality. The varying association of cognitive impairment and all-cause mortality in different age groups might be due to the survival bias that the oldest old represent hardy survivors who have successfully adapted to cognitive impairment [31]. Another possible explanation is that the oldest old have a higher risk of mortality, a common competing risk for cognitive impairment, thus causing loss to follow up bias and confusing the association [13].

Several strengths are worth mentioning in our findings. We included the representative sample to explore the relationship between cognitive impairment, evaluated by different cut-offs, and mortality among the Chinese elderly population based on two 6-year cohorts. Moreover, our age-specific analyses including 65-79, 80-89, 90-99 and $\geq$ 100 age groups can help us obtain a more comprehensive understanding of the impact of cognitive impairment on risk of mortality in elders. In addition, our sensitivity analyses suggested that the findings of this study were robust.

Some limitations of this study should be acknowledged. Firstly, cognitive impairment was measured using the MMSE (not based on clinical diagnosis) and we were not being able to distinguish between elders with and without dementia. However, we tried to reduce the impact of long-term progression about cognitive function by tested the interaction cognitive impairment and functional impairment. Secondly, despite the effort of adjusting for a number of confounders such as demographic characteristics, lifestyle factors, heath conditions, health service and economic status, we could not completely eliminate the risk of confounding bias due to unobserved differences in personal characteristics. Thirdly, the association of rapid cognitive decline and mortality might differ in the elderly whose cognitive status did not decline or declined slowly. In our study, we only focused on baseline cognitive impairment and did not assess whether cognitive decline over time was associated with elevated risk of mortality.

\section{Conclusions}

The data from this population-based longitudinal study revealed that cognitive impairment was significantly 
associated with increased risk of all-cause mortality, and the relationship of cognitive impairment, stratified by educational levels with mortality was lower than previous studies. Thus, prevention and management of cognitive impairment taking the educational levels into account might have substantial benefits for mortality in the health policies or clinical practice. .

\section{Supplementary information}

Supplementary information accompanies this paper at https://doi.org/10. 1186/s12877-020-1424-4.

Additional file 1: Figure S1. Kaplan-Meier curve for hazard of death by the baseline MMSE score (Left: 2002-2008, Right: 2008-2014). Table S1. The subgroup analyses of hazard ratios $(95 \% \mathrm{Cl})$ for all-cause mortality according to baseline MMSE score. Table S2. Association of combined cognition-ADL function with all-cause mortality among elders in the 2002-2008 and 2008-2014. Table S3. Sensitivity analyses for the association between cognitive impairment $(M M S E<24)$ and all-cause mortality.

Table S4. Sensitivity analyses for the association between cognitive impairment ( taking into account the educational background) and allcause mortality.

\section{Abbreviations}

ADL: Activities of Daily Living; Cls: Confidence Intervals; CLHLS: Chinese Longitudinal Healthy Longevity Survey; HRs: Hazard Ratios; MMSE: MiniMental State Examination

\section{Acknowledgments}

We are grateful to all participating people and their families. We acknowledge all staffs for their great contributions to the success of the program.

\section{Authors' contributions}

$\mathrm{JD}$ and $\mathrm{YBL}$ conducted the data analysis, drafted and revised the manuscript. $X M S, H S$ and $Y Z$ designed the survey, reviewed, and revised this manuscript. $X G$ and VBK helped to conduct data analysis and critically revised this manuscript, JHZ helped to implement the survey, and review the manuscript. All authors have read and approved the final manuscript.

\section{Funding}

This work was supported by the Chinese Longitudinal Healthy Longevity Survey, which provided the data in this article, is jointly supported by National Natural Sciences Foundation of China $(81,273,160$ and 81,573,247). The funder of the National Natural Sciences Foundation of China (81273160) played role in study design or implementation, data collection, management, analysis, and interpretation, manuscript preparation, review, or approval; or the decision to submit the manuscript for publication.

\section{Availability of data and materials}

This study was based on the datasets from the Chinese Longitudinal Healthy Longevity Survey (CLHLS) in longevity areas. The CLHLS data can be publicly obtained through the National Archive of Computerized Data on Aging (NACDA).

(https://www.icpsr.umich.edu/icpsrweb/NACDA/series/487)

\section{Ethics approval and consent to participate}

Written consent was obtained from all participants and/or their relatives, and the study was approved by the Ethics Committee of Peking University (IRB00001052-13074).

\section{Consent for publication}

Not applicable.

\section{Competing interests}

The authors declare that they have no competing interests.

\section{Author details}

${ }^{1}$ Department of Epidemiology and Health Statistics, School of Public Health, Anhui Medical University, 81 Meishan Road, Hefei 230032, Anhui Province, China. ${ }^{2}$ National Institute of Environmental Health, Chinese Center for Disease Control and Prevention, \#7 Panjiayuan Nanli, Chaoyang, Beijing 100021, China. ${ }^{3}$ Nutritional Epidemiology Lab, Pennsylvania State University, Philadelphia, PA, USA. ${ }^{4}$ Duke Molecular Physiology Institute and Division of Rheumatology, Department of Medicine, Duke University School of Medicine, Durham, NC, USA. ${ }^{5}$ Center for the study of Aging and Human Development and the Geriatric Division of School of Medicine, Duke University, Durham, NC, USA. ${ }^{6}$ Center for Study of Healthy Aging and Development Studies, Peking University, Beijing, China.

Received: 17 September 2019 Accepted: 13 January 2020

Published online: 28 January 2020

\section{References}

1. Langa KM, Larson EB, Karlawish JH, et al. Trends in the prevalence and mortality of cognitive impairment in the United States: is there evidence of a compression of cognitive morbidity? Alzheimers Dement. 2008;4:134-44. https://doi.org/10.1016/j.jalz.2008.01.001.

2. Hao Q, Dong $B$, Yang $M$, et al. Frailty and cognitive impairment in predicting mortality among oldest-old people. Front Aging Neurosci. 2018; 10:295. https://doi.org/10.1007/s12603-011-0096-3.

3. Perna L, Wahl HW, Mons U, et al. Cognitive impairment, all-cause and cause-specific mortality among non-demented older adults. Age Ageing. 2015;44:445-51. https://doi.org/10.1093/ageing/afu188.

4. Jia J, Wang F, Wei $C$, et al. The prevalence of dementia in urban and rural areas of China. Alzheimers Dement. 2014;10:1-9. https://doi.org/10.1016/j. jalz.2013.01.012.

5. Ng TP, Feng L, Nyunt $M$, et al. Metabolic syndrome and the risk of mild cognitive impairment and progression to dementia. Jama Neurol. 2016;73: 456-63. https://doi.org/10.1001/jamaneurol.2015.4899.

6. Jia J, Zhou A, Wei C, et al. The prevalence of mild cognitive impairment and its etiological subtypes in elderly Chinese. Alzheimers Dement. 2014;10:43947. https://doi.org/10.1016/j.jalz.2013.09.008.

7. Georgakis MK, Papadopoulos FC, Protogerou AD, et al. Comorbidity of cognitive impairment and late-life depression increase mortality. J Geriatr Psych Neur. 2016;29:195-204. https://doi.org/10.1177/0891988716632913.

8. Hapca S, Guthrie B, Cvoro V, et al. Mortality in people with dementia, delirium, and unspecified cognitive impairment in the general hospital: prospective cohort study of 6,724 patients with 2 years follow-up. Clin Epidemiol. 2018;10:1743-53. https://doi.org/10.2147/CLEP.S174807.

9. Downer B, Al Snih S, Howrey BT, et al. Combined effects of cognitive impairment and pre-frailty on future frailty and death in older Mexican Americans. Aging Ment Health. 2018:1-8. https://doi.org/10.1080/13607863. 2018.1493719

10. Gombojav B, Yi S, Sull JW, et al. Combined effects of cognitive impairment and hypertension on Total mortality in elderly people: the Kangwha cohort study. Gerontology. 2011;57:490-6. https://doi.org/10.1159/000323759.

11. Ablett AD, McCarthy K, Carter B, et al. Cognitive impairment is associated with mortality in older adults in the emergency surgical setting: findings from the older persons surgical outcomes collaboration (OPSOC): a prospective cohort study. Surgery. 2018. https://doi.org/10.1016/j.surg.2018.10.013.

12. Griva K, Stygall J, Hankins M, et al. Cognitive impairment and 7-year mortality in Dialysis patients. Am J Kidney Dis. 2010;56:693-703. https://doi. org/10.1053/j.ajkd.2010.07.003.

13. An R, Liu G. Cognitive impairment and mortality among the oldest-old Chinese. Int J Geriatr Psychiatry. 2016;31:1345-53. https://doi.org/10.1002/gps.4442.

14. Gao S, Jin Y, Unverzagt FW, et al. Cognitive function, body mass index and mortality in a rural elderly Chinese cohort. Arch Public Health. 2014;72:9. https://doi.org/10.1186/2049-3258-72-9.

15. Bravo G. Réjean Hébert. Age- and education-specific reference values for the mini-mental and modified mini-mental state examination derived from a non-demented elderly population. Int J Geriatric Psychiatry. 1997;12(10): 1008-18. https://doi.org/10.1002/(sici)1099-1166(199710)12:10<1008:.:aidgps676>3.0.co;2-a.

16. Cui G, Yao Y, Xu R, et al. Cognitive impairment using education-based cutoff points for CMMSE scores in elderly Chinese people of agricultural and rural Shanghai China. Acta Neurol Scand. 2011;124(6). https://doi.org/10. 1111/j.1600-0404.2010.01484.x. 
17. An J, Li H, Tang Z, et al. Cognitive impairment and risk of all-cause and cardiovascular disease mortality over 20-year follow-up: results from the BLSA. J Am Heart Assoc. 2018;7. https://doi.org/10.1161/JAHA.117.008252.

18. Zeng $Y$, Feng $Q$, Hesketh $T$, et al. Survival, disabilities in activities of daily living, and physical and cognitive functioning among the oldest-old in China: a cohort study. Lancet. 2017;389:1619-29. https://doi.org/10.1016/ S0140-6736(17)30548-2.

19. Zeng $Y$. Towards deeper research and better policy for healthy aging-using the unique data of Chinese longitudinal healthy longevity survey. Chin Economic J. 2012;5:131-49. https://doi.org/10.1080/17538963.2013.764677.

20. Christensen K, Thinggaard M, Oksuzyan A, et al. Physical and cognitive functioning of people older than 90 years: a comparison of two Danish cohorts born 10 years apart. Lancet. 2013;382:1507-13. https://doi.org/10. 1016/50140-6736(13)60777-1.

21. Zeng Y, Vaupel JW. Functional capacity and self-evaluation of health and life of oldest old in China. J Soc Issues. 2002;58:733-48. https://doi.org/10.1111/ 1540-4560.00287.

22. Schultz-Larsen K, Rahmanfard N, Kreiner S, et al. Cognitive impairment as assessed by a short form of MMSE was predictive of mortality. I Clin Epidemiol. 2008;61:1227-33. https://doi.org/10.1016/j.jclinepi.2007.12.007.

23. Yuan J, LV Y, Chen $H$, et al. Association between late-life blood pressure and the incidence of cognitive impairment: a community-based prospective cohort study. J Am Med Dir Assoc. 2019;20:177-82. https://doi.org/10.1016/j. jamda.2018.05.029.

24. Zhang MY, Katzman R, Salmon D, et al. The prevalence of dementia and Alzheimer's disease in Shanghai, China: impact of age, gender and education. Ann Neurol. 1990;27:428-37. https://doi.org/10.1002/ana.410270412.

25. Li ZH, Lv YB, Kraus VB, et al. Trends in the Incidence of Activities of Daily Living Disability Among Chinese Elderly from 2002-2014, J Gerontol A Biol Sci Med Sci. 2019. https://doi.org/10.1093/gerona/glz221.

26. Lee Y, Kim J, Chon D, et al. The effects of frailty and cognitive impairment on 3-year mortality in older adults. Maturitas. 2018;107:50-5. https://doi.org/ 10.1016/j.maturitas.2017.10.006.

27. Yu W, Chou M, Peng L, et al. Synergistic effects of cognitive impairment on physical disability in all-cause mortality among men aged 80 years and over: results from longitudinal older veterans study. PLoS One. 2017;12: e181741. https://doi.org/10.1371/journal.pone.0181741.

28. Kane KD, Yochim BP, Lichtenberg P. A. Depressive symptoms and cognitive impairment predict all-cause mortality in long-term care residents. Psychol Aging. 2010;25:446-52. https://doi.org/10.1037/a0019032.

29. Farid $K$, Zhang $Y$, Bachelier $D$, et al. Cognitive impairment and malnutrition, predictors of all-cause mortality in hospitalized elderly subjects with cardiovascular disease. Arch Cardiovasc Dis. 2013;106:188-95. https://doi. org/10.1016/j.acvd.2012.12.006.

30. Yu H. Universal health insurance coverage for 1.3 billion people: what accounts for China's success? Health Policy. 2015;119:1145-52. https://doi. org/10.1016/j.healthpol.2015.07.008.

31. Bassuk S, Wypij D, Berkman LF. Cognitive impairment and mortality in the community-dwelling elderly. Am J Epidemiol. 2000;151:676-88. https://doi. org/10.1080/09540120050001896.

32. Hai S, Cao L, Yang X, et al. Association between nutrition status and cognitive impairment among Chinese nonagenarians and centenarians. Int J Gerontol. 2017;11:215-9. https://doi.org/10.1016/j.ijge.2016.12.002.

33. Wu J, Liu J, Zhu B, et al. Does China's new medical reform improve health equity of rural residents? Evidence from household surveys before and after the implementation of new medical reform in Shanxi Province, China. Value Health. 2015;18:A526. https://doi.org/10.1016/j.jval.2015.09.1623.

34. Drew DA, Weiner DE, Tighiouart $H$, et al. Cognitive function and all-cause mortality in maintenance hemodialysis patients. Am J Kidney Dis. 2015;65: 303-11. https://doi.org/10.1053/j.ajkd.2014.07.009.

35. Nybo H, Petersen HC, Gaist D, et al. Predictors of mortality in 2,249 nonagenarians-the Danish 1905 cohort survey. J Am Geriatr Soc. 2003;51: 1365-73. https://doi.org/10.1046/j.1532-5415.2003.51453.x.

36. Gale CR, Martyn CN, Cooper C. Cognitive impairment and mortality in a cohort of elderly people. BMJ. 1996;312:608-11. https://doi.org/10.1136/bmj. 312.7031.608.

\section{Publisher's Note}

Springer Nature remains neutral with regard to jurisdictional claims in published maps and institutional affiliations.

Ready to submit your research? Choose BMC and benefit from:

- fast, convenient online submission

- thorough peer review by experienced researchers in your field

- rapid publication on acceptance

- support for research data, including large and complex data types

- gold Open Access which fosters wider collaboration and increased citations

- maximum visibility for your research: over $100 \mathrm{M}$ website views per year

At BMC, research is always in progress.

Learn more biomedcentral.com/submissions 